\title{
Leptospirosis as Unusual Trigger of Systemic Lupus Erythematosus
}

\author{
Jinhee Lee, Yang Ree Kim, Chong-Hyeon Yoon \\ Department of Internal Medicine, Uijeongbu St. Mary's Hospital, College of Medicine, The Catholic University of Korea, Seoul, Korea
}

\begin{abstract}
Systemic lupus erythematosus (SLE) is a chronic autoimmune disorder of an unknown origin. The role of leptospirosis as a triggering factor for SLE is unknown. This paper reports an uncommon case of SLE following a leptospira infection. A 29-year-old female was referred due to fevers, myalgia, and facial edema with rash. Laboratory investigations revealed a hepatic dysfunction, significantly raised lactate dehydrogenase with marked leukopenia and thrombocytopenia. A diagnosis of leptospirosis was confirmed. The patient was treated with antibiotic therapy for leptospirosis. She developed dyspnea after one week. The echocardiogram revealed global hypokinesia with a decreased ejection fraction. A positivity of antinuclear, anti-DNA, and anti-Smith antibodies, together with clinical and laboratory improvement by steroid therapy, led to the diagnosis of SLE. This case highlights the presence of concurrent SLE and leptospirosis. As the symptoms of SLE are similar to leptospirosis, accurate diagnosis through high suspicion is essential for appropriate treatment. (J Rheum Dis 2019;26:79-82)
\end{abstract}

Key Words. Systemic lupus erythematosus, Leptospirosis, Myocarditis

\section{INTRODUCTION}

Systemic lupus erythematosus (SLE) is a chronic autoimmune disorder with an unknown origin [1]. Many researchers have focused on the role of infection in the pathogenesis of SLE, particularly infections caused by viruses, bacteria, and parasites [2]. The mechanisms, however, remain poorly understood. Some studies have shown that autoimmune diseases, such as Guillain-Barré syndrome [3], autoimmune hepatitis [4], and autoimmune epilepsy [5], were a result of leptospirosis. On the other hand, the role of leptospirosis as a triggering factor for SLE is unknown.

Leptospirosis is a zoonosis caused by the spirochetes of the genus Leptospira. Transmission may follow direct contact with soil or water contaminated with the urine of infected rodents [6]. Leptospirosis is a biphasic illness characterized by an early septicemic phase and a delayed immune phase. The clinical spectrum of the disease rang- es from self-limited febrile episodes to severe organ dysfunction. This paper reports an uncommon case of newly diagnosed SLE during the treatment of leptospirosis.

\section{CASE REPORT}

A 29-year-old woman visited the emergency department with fever, myalgia, and facial edema with a rash since the previous 7 days during late summer. She had no significant prior medical history. The facial rash was a nonpruritic, erythematous patch with multiple crust on the nose and cheeks, and diffuse swelling was observed on the whole face. Two weeks before the onset of illness, the patient traveled to the riverside. Her temperature was $38.9^{\circ} \mathrm{C}$, pulse 107 beats $/ \mathrm{min}$, blood pressure 95/70 $\mathrm{mmHg}$, and respiratory rate 25 breaths/min. The laboratory data revealed the following: white blood cell (WBC) $1.000 \times 10^{9} / \mathrm{L}$, hemoglobin $8.7 \mathrm{~g} / \mathrm{dL}$, platelet $86 \times 10^{9} / \mathrm{L}$, erythrocyte sedimentation rate $19 \mathrm{~mm} / \mathrm{h}$

Received : May 8, 2018, Revised : June 1, 2018, Accepted : June 9, 2018

Corresponding to : Chong-Hyeon Yoon iDhttp://orcid.org/0000-0003-2305-4637

Division of Rheumatology, Department of Internal Medicine, Uijeongbu St. Mary's Hospital, College of Medicine, The Catholic University of Korea, 271 Cheonbo-ro, Uijeongbu 11765, Korea. E-mail : chyoon@catholic.ac.kr

Copyright (c) 2019 by The Korean College of Rheumatology. All rights reserved.

This is a Open Access article, which permits unrestricted non-commerical use, distribution, and reproduction in any medium, provided the original work is properly cited. 
(normal 0 10), C-reactive protein (CRP) $0.23 \mathrm{mg} / \mathrm{dL}$ (normal <0.3), procalcitonin $0.385 \mathrm{ng} / \mathrm{mL}$ (normal $<0.046$ ), aspartate aminotransferase (AST) $570 \mathrm{U} / \mathrm{L}$, alanine aminotransferase (ALT) $516 \mathrm{U} / \mathrm{L}$, lactate dehydrogenase (LDH) 1,213 U/L, and creatine phosphokinase (CPK) $246 \mathrm{U} / \mathrm{L}$. Urinalysis showed protein $1+$ and few bacteria. The chest X-ray showed a normal heart size and lung parenchyma. The electrocardiography (ECG) was normal. The chest and abdominal computed tomography (CT) scan revealed no significant abnormal findings. The paranasal sinus CT scan showed diffuse swelling of the face with a streaky infiltration in the sub-

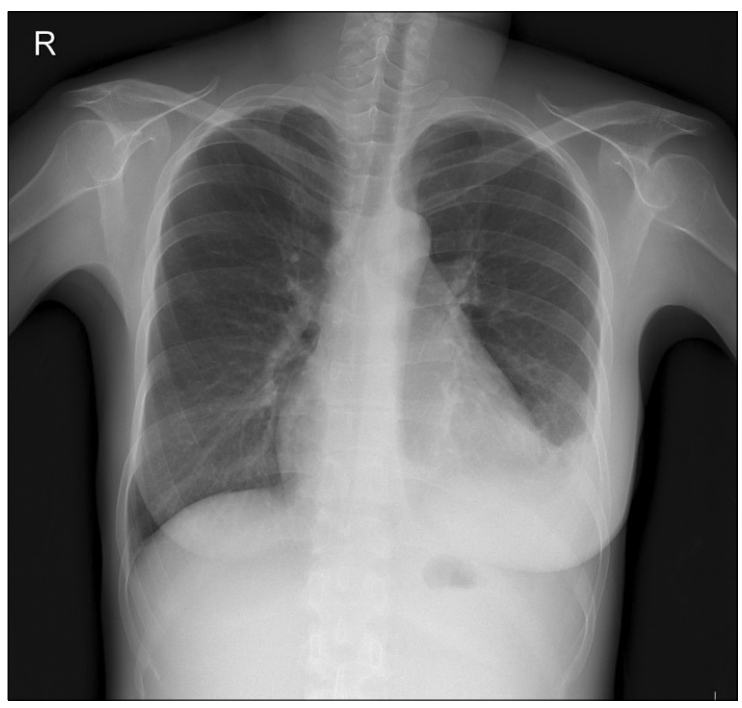

Figure 1. Chest X-ray showed cardiomegaly with left-sided pleural effusion on the 13th day of illness. cutaneous fat layer. The patient was immediately commenced on empirical intravenous antibiotics with piperacillin/tazobactam for presumed facial cellulitis. On the 4th day, her fever improved but the leukopenia, thrombocytopenia and elevated liver enzymes persisted. A viral examination revealed negative serology for hepatitis A, B, $C$, herpes simplex virus, human immunodeficiency virus, cytomegalovirus, Epstein-Barr virus, and parvovirus. The antibodies to leptospira were positive in 1:160 by the microscopic agglutination test (MAT) method. The patient was suspected of leptospirosis and doxycycline was added. One week later, the measured level of antibodies to leptospira was $1: 1,280$, which was more than four times higher than the first, confirming leptospirosis. Despite the combinations of antibiotic therapy, the laboratory abnormalities were not improved. On the 13th day, she suddenly complained of shortness of breath and chest tightness. The facial rash was persisted. The laboratory data showed WBC 2,120 $\times 10^{9} / \mathrm{L}$, hemoglobin $10.2 \mathrm{~g} / \mathrm{dL}$, platelet $45 \times 10^{9} / \mathrm{L}, \mathrm{CRP} 0.21 \mathrm{mg} / \mathrm{dL}$, AST $675 \mathrm{U} / \mathrm{L}$, ALT $167 \mathrm{U} / \mathrm{L}, \mathrm{LDH} 1,626 \mathrm{U} / \mathrm{L}$, and CPK 2,503 U/L. Urinalysis was normal. The chest X-ray showed cardiomegaly with left-sided pleural effusion (Figure 1). ECG showed a sinus rhythm with a $\mathrm{T}$ wave inversion in leads II, III, and V2 V6 (Figure 2A). The cardiac enzyme showed CK-MB $10.28 \mathrm{ng} / \mathrm{mL}$ (normal $0.2 \sim 2.88$ ), troponin-I 0.063 $\mathrm{ng} / \mathrm{mL}$ (normal <0.78), and pro-BNP $6,268 \mathrm{pg} / \mathrm{mL}$ (normal 5 125). Echocardiography revealed global hypokinesia with a left ventricular ejection fraction of $42 \%$ and a moderate amount of pericardial effusion. The antinuclear antibody was positive in 1:1,600 titers. The an-

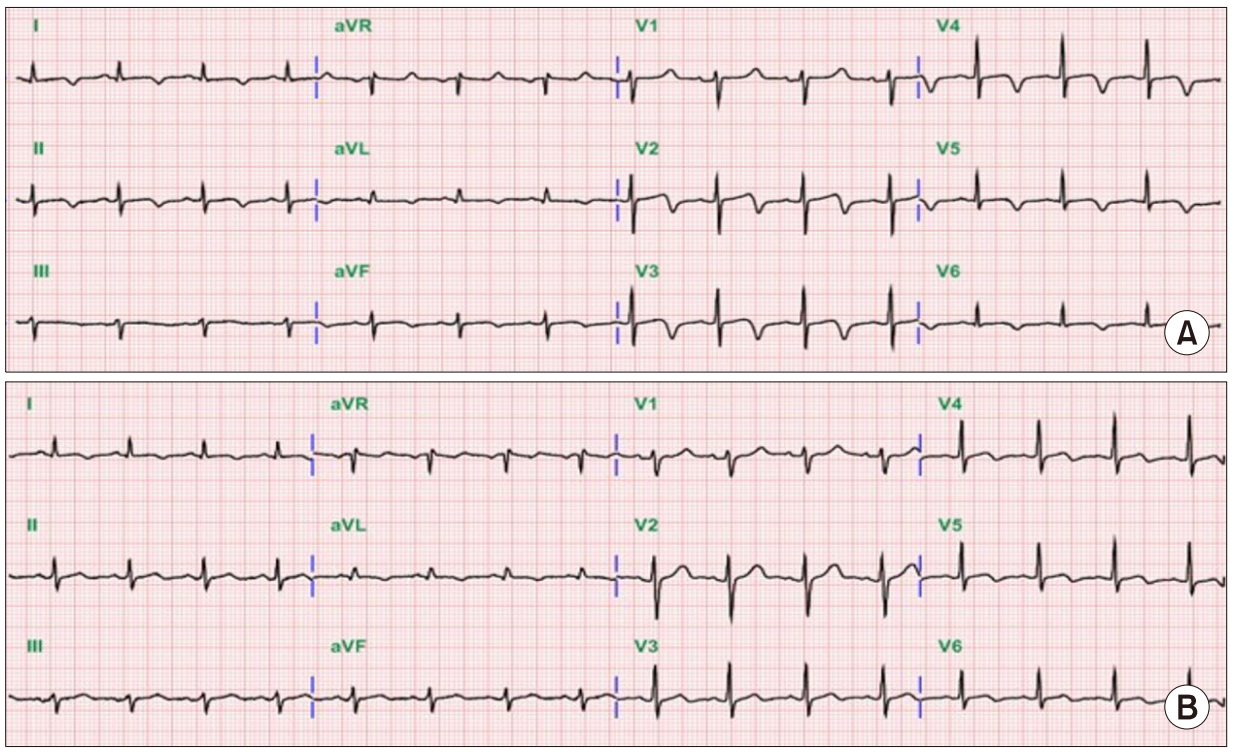

Figure 2. (A) Electrocardiogram showing a normal sinus rhythm with $T$ wave inversion in leads II, III and V2 to V6. (B) After the prednisolone treatment, the electrocardiogram showed a normal sinus rhythm with normally positive T wave. 
ti-dsDNA, anti-Ro/La, anti-Smith, and anti-ribosomal P antibodies were positive. The complement levels were low (C3 $13 \mathrm{mg} / \mathrm{dL}$ [normal 76 139]; C4, $2.7 \mathrm{mg} / \mathrm{dL}$ [normal 12 37]) and the direct Coomb's test was positive. She fulfilled the Systemic Lupus International Collaborating Clinics criteria for a diagnosis of SLE [7]. Therefore, she was diagnosed with SLE concomitant with leptospirosis. The left ventricular dysfunction was believed to be caused by the SLE flare, and treatment with 1 $\mathrm{mg} / \mathrm{kg} /$ day of prednisolone was begun. The shortness of breath, liver dysfunction, and pancytopenia improved after 1 week of prednisolone treatment. The abnormal findings on ECG and chest X-ray were returned to normal (Figure 2B). The echocardiography conducted 2 weeks after prednisolone therapy showed a normal left ventricular ejection fraction without pericardial effusion. The patient was discharged with $60 \mathrm{mg}$ of prednisolone. After discharge, the dose of prednisolone was reduced gradually to $5 \mathrm{mg}$ for 5 months. After one month, leukopenia, hair loss and facial rash occurred. The dose of prednisolone was increased to $20 \mathrm{mg}$ and her symptoms improved. The dose of steroid was then tapered. The patient was on medication with prednisolone $5 \mathrm{mg}$ and hydroxychloroquine in the outpatient clinic.

\section{DISCUSSION}

Leptospirosis has been reported worldwide, particularly during the rainy season in late summer or early autumn [6]. The incubation period is usually $1 \sim 2$ weeks. A confirmatory diagnosis is based on isolation of the organism, on a positive result in the polymerase chain reaction, or on seroconversion or fourfold or greater between the acute and convalescent specimens in the MAT method [8]. In cases with strong clinical evidence of leptospirosis, a single antibody titer of $>1: 800$ is evidence of a current or recent infection.

In the present case, the early symptoms were fever, myalgia, and facial edema with rash. Flu-like illness, such as fever and myalgia, might be considered the presentation of the septicemic phase of leptospirosis. The patient's fever and myalgia improved during antibiotics therapy but facial rash was not changed. In addition, proteinuria, which was observed at admission, was not detected after the antibiotic treatment. Renal involvement in leptospirosis is common and proteinuria, pyuria, and microscopic hematuria are observed frequently in urinalysis [8]. According to the patient's travel history, the timing of symptoms and response to antibiotics, fever and proteinuria are more likely to be related to leptospirosis.

The patient had an erythematous rash with multiple crust on the nose and both cheeks and diffuse swelling on the whole face. In leptospirosis, the nonspecific macular rash can be seen and is usually not severe, transient and not itchy $[9,10]$. The patient's rash was not consistent with the typical patterns observed in leptospirosis. A lupus rash is a butterfly-shaped rash that appears over the bridge of the nose and cheeks. Facial edema with crust accompanied by erythematous rash has rarely been reported in SLE [11]. In the present case, the patient's rash improved drastically after prednisolone therapy. Therefore, the patient's facial rash is considered to be a presentation of SLE.

At the time of admission, her symptoms were considered to be infectious disease, but CRP was normal. According to Crouzet et al. [12], high CRP levels are observed in most patients of leptospirosis. On the other hand, elevated CRP was $67 \% \sim 75 \%$ in a retrospective study of leptospirosis [13]. Therefore, a normal or only slightly elevated CRP does not exclude a leptospirosis infection.

The most severe clinical manifestation in this case was left ventricular dysfunction due to myocarditis. Myocarditis is an immune-inflammatory state of the myocardium [14]. In many studies, myocarditis associated with leptospirosis is rare and is improved by antibiotics therapy [15]. In the present case, the clinical and laboratory findings deteriorated despite the antibiotics therapy but improved rapidly after prednisolone therapy. Therefore, myocarditis is considered to have developed by SLE rather than leptospirosis.

In conclusion, this paper reported a case of newly diagnosed SLE during the treatment of leptospirosis. As SLE and leptospirosis share similar clinical features, accurate diagnosis through high suspicion is essential for appropriate treatment. Leptospirosis may be considered a rare cause of SLE, but further studies will be needed to determine the role of leptospirosis as a trigger factor for SLE.

\section{SUMMARY}

This case highlights the presence of concurrent SLE and leptospirosis. As the symptoms of SLE are similar to leptospirosis, accurate diagnosis through high suspicion is 
essential for appropriate treatment.

\section{CONFLICT OF INTEREST}

No potential conflict of interest relevant to this article was reported.

\section{REFERENCES}

1. Laxminarayana D. Molecular insights into systemic lupus erythematosus pathogenesis. Clin Med Insights Pathol 2014;20;7:7-9.

2. Esposito S, Bosis S, Semino M, Rigante D. Infections and systemic lupus erythematosus. Eur J Clin Microbiol Infect Dis 2014;33:1467-75.

3. Bal AM, Bharadwaj RS, Gita N, Joshi SA, Thakare JP. Guillain-Barre syndrome in a pediatric patient following infection due to Leptospira. Jpn J Infect Dis 2003;56:29-31.

4. Urganci N, Kalyoncu D, Cayonu N, Erdem E, Yildirmak Y, Yilmaz B. Acute liver failure, autoimmune hepatitis, and leptospirosis: a case report. Pediatr Emerg Care 2011;27:963-5.

5. Makhija P, Gopinath S, Kannoth S, Radhakrishnan K. A case of post-leptospirosis autoimmune epilepsy presenting with sleep-related hypermotor seizures. Epileptic Disord 2017; 19:456-60.

6. Levett PN. Leptospirosis. Clin Microbiol Rev 2001;14: 296-326.

7. Petri M, Orbai AM, Alarcón GS, Gordon C, Merrill JT, Fortin $\mathrm{PR}$, et al. Derivation and validation of the systemic lupus in- ternational collaborating clinics classification criteria for systemic lupus erythematosus. Arthritis Rheum 2012;64: 2677-86.

8. Budihal SV, Perwez K. Leptospirosis diagnosis: competancy of various laboratory tests. J Clin Diagn Res 2014;8: 199-202.

9. Puca E, Pilaca A, Kalo T, Pipero P, Bino S, Hysenaj Z, et al. Ocular and cutaneous manifestation of leptospirosis acquired in Albania: a retrospective analysis with implications for travel medicine. Travel Med Infect Dis 2016;14:143-7.

10. Barnabe C, Fahlman N. Overlapping clinical features of lupus and leptospirosis. Clin Rheumatol 2008;27 Suppl 1:S23-5.

11. Castro LA, Davis DM, Davis MD, Bruce AJ, Pittelkow MR. Facial edema and crusted patches: a precursor to life-threatening acute systemic lupus erythematosus. J Am Acad Dermatol 2007;56(5 Suppl):S126-7.

12. Crouzet J, Faucher JF, Toubin M, Hoen B, Estavoyer JM. Serum C-reactive protein (CRP) and procalcitonin (PCT) levels and kinetics in patients with leptospirosis. Eur J Clin Microbiol Infect Dis 2011;30:299-302.

13. Mori M, VAN Esbroeck M, Depoorter S, Decaluwe W, Vandecasteele SJ, Fretin D, et al. Outbreak of leptospirosis during a scout camp in the Luxembourg Belgian province, Belgium, summer 2012. Epidemiol Infect 2015;143:1761-6.

14. Navinan MR, Rajapakse S. Cardiac involvement in leptospirosis. Trans R Soc Trop Med Hyg 2012;106:515-20.

15. Trivedi SV, Bhattacharya A, Amichandwala K, Jakkamsetti V. Evaluation of cardiovascular status in severe leptospirosis. J Assoc Physicians India 2003;51:951-3. 